\title{
Cohomology classes of rank varieties and a counterexample to a conjecture of Liu
}

\author{
Brendan Pawlowskil| \\ Department of Mathematics, University of Minnesota, Minneapolis, MN, USA
}

\begin{abstract}
To each finite subset of a discrete grid $\mathbb{N} \times \mathbb{N}$ (a diagram), one can associate a subvariety of a complex Grassmannian (a diagram variety), and a representation of a symmetric group (a Specht module). Liu has conjectured that the cohomology class of a diagram variety is represented by the Frobenius characteristic of the corresponding Specht module. We give a counterexample to this conjecture.

However, we show that for the diagram variety of a permutation diagram, Liu's conjectured cohomology class $\sigma$ is at least an upper bound on the actual class $\tau$, in the sense that $\sigma-\tau$ is a nonnegative linear combination of Schubert classes. To do this, we consider a degeneration of Coskun's rank varieties which contains the appropriate diagram variety as a component. Rank varieties are instances of Knutson-Lam-Speyer's positroid varieties, whose cohomology classes are represented by affine Stanley symmetric functions. We show that the cohomology class of a rank variety is in fact represented by an ordinary Stanley symmetric function.
\end{abstract}

Résumé. À chaque sous-ensemble fini de $\mathbb{N} \times \mathbb{N}$ (un diagramme), on peut associer une sous-variété d'une grassmannienne complexe et une représentation d'un groupe symétrique (un module de Specht). Liu a conjecturé que la classe de cohomologie de la variété d'un diagramme est représentée par la caractéristique de Frobenius du module de Specht correspondant. Nous donnons un contre-exemple à cette conjecture.

Cependant, nous montrons que dans le cas de la variété du diagramme de permutation, la classe de cohomologie conjecturée par Liu est au moins un majorant de la classe juste $\tau$, c'est-à-dire que $\sigma-\tau$ est une combinaison linéaire non-négative des classes de Schubert. Pour ce faire, nous considérons une dégénérescence des variétés de rang de Coskun qui contient la variété appropriée d'un diagramme comme une composante irréductible. Les variétés de rang sont des exemples de variétés de positrö̈de, dont les classes de cohomologie sont représentées par des fonctions symétriques de Stanley affines. En effet, nous montrons que la classe de cohomologie d'une variété de rang est représentée par une fonction symétrique de Stanley ordinaire.

Keywords: Specht modules, Schubert calculus, positroid varieties, Stanley symmetric functions

\section{Introduction}

A finite subset $D$ of $\mathbb{N} \times \mathbb{N}$ is called a diagram. Write $[n]$ for $\{1,2, \ldots, n\}$. One can associate a complex representation $S^{D}$ of the symmetric group $S_{|D|}$ to a diagram $D$, called the Specht module of $D$. These

\footnotetext{
${ }^{\dagger}$ Partially supported by DMS-1101017 from the NSF.

1365-8050 @ 2015 Discrete Mathematics and Theoretical Computer Science (DMTCS), Nancy, France
} 
generalize the usual irreducible Specht modules, which occur when $D$ is the Young diagram of a partition; the definition for general diagrams appears in James and Peel (1979). Write $s_{D}$ for the Frobenius characteristic of $S^{D}$.

Let $\Lambda$ be the ring of symmetric functions over $\mathbb{Z}$, and $\operatorname{Gr}(k, n)$ the Grassmannian variety of $k$-planes in $\mathbb{C}^{n}$. It is well known that the cohomology ring $H^{*}(\operatorname{Gr}(k, n), \mathbb{Z})$ is a quotient of $\Lambda$. Specifically, the Schubert varieties $X_{\lambda}$ in $\operatorname{Gr}(k, n)$, indexed by partitions fitting inside the rectangle $[k] \times[n-k]$, give a cell decomposition of $\operatorname{Gr}(k, n)$ (in the classical topology) with only even-dimensional cells. The cohomology classes $\sigma_{\lambda}=\left[X_{\lambda}\right]$, called Schubert classes, then form a basis of $H^{*}(\operatorname{Gr}(k, n))$. Define a surjective linear map $\phi: \Lambda \rightarrow H^{*}(\operatorname{Gr}(k, n))$ by

$$
\phi\left(s_{\lambda}\right)=\left\{\begin{array}{ll}
\sigma_{\lambda} & \lambda \subseteq[k] \times[n-k] \\
0 & \text { else }
\end{array} .\right.
$$

This map turns out to be a ring homomorphism, meaning that intersection product computations in Grassmannians can be done in terms of symmetric functions.

A natural problem is to start with a homogeneous Schur-positive symmetric function $f$ and ask for an irreducible subvariety of $\operatorname{Gr}(k, n)$ with cohomology class $\phi(f)$. Liu's conjecture concerns this problem for Schur functions of diagrams.

Definition 1.1 Given a diagram $D$ contained in $[k] \times[n-k]$, the associated open diagram variety is

$$
X_{D}^{\circ}=\left\{\operatorname{rowspan}[A \mid I]: A \in M_{k, n-k} \text { with } A_{i j}=0 \text { whenever }(i, j) \in D\right\},
$$

where $M_{k, n-k}$ is the set of $k \times(n-k)$ complex matrices and I is the $k \times k$ identity matrix. The diagram variety $X_{D}$ of $D$ is then the closure of $X_{D}^{\circ}$ in the Zariski topology.

Example 1.2 We will draw diagrams in matrix coordinates (with $(1,1)$ in the upper left), using $\circ$ for those lattice points in the diagram (which we call cells) and ·for the others, e.g.

$$
D=\begin{array}{ccc}
\cdot & \circ & \circ \\
\circ & \circ & \circ
\end{array}
$$

Then $X_{D}$ in $\operatorname{Gr}(3,6)$ is the closure of

$$
\left\{\operatorname{rowspan}\left[\begin{array}{llllll}
0 & * & * & 1 & 0 & 0 \\
* & 0 & 0 & 0 & 1 & 0 \\
0 & 0 & * & 0 & 0 & 1
\end{array}\right]\right\} .
$$

Since $X_{D}^{\circ}$ is isomorphic to affine space of dimension $k(n-k)-|D|, X_{D}$ is irreducible of codimension $|D|$.

Conjecture 1 (Liu (2010)) For any diagram $D$, the cohomology class $\left[X_{D}\right]$ is $\phi\left(s_{D}\right)$.

Liu proved Conjecture 2, or the weaker variant claiming equality of degrees, for various classes of diagrams. However, it turns out that this conjecture fails in general, as we show in Section 2

Theorem 1.3 If $D=\{(1,1),(2,2),(3,3),(4,4)\}, k=4, n=8$, then Conjecture 2 is false. 
In some cases, we can at least show that $\phi\left(s_{D}\right)$ gives an upper bound for $\left[X_{D}\right]$. The diagram $D(w)$ of a permutation $w$ (sometimes Rothe diagram) has one cell $(i, w(j))$ for each inversion $i<j, w(i)>w(j)$ of $w$.

Theorem 1.4 For any permutation $w, \phi\left(s_{D(w)}\right)-\left[X_{D(w)}\right]$ is a nonnegative linear combination of Schubert classes.

In Reiner and Shimozono (1995b) (and essentially in Kraśkiewicz and Pragacz (2004)) it is shown that for a permutation $w \in S_{n}, s_{D(w)}$ is actually the Stanley symmetric function $F_{w}$ (or $F_{w^{-1}}$, depending on conventions). Building on Postnikov (2006), Knutson et al. (2013) have defined a collection of irreducible subvarieties $\Pi_{f}$ of Grassmannians called positroid varieties, indexed by certain affine permutations $f$. They show that the cohomology class of $\Pi_{f}$ is $\phi\left(\tilde{F}_{f}\right)$, where $\tilde{F}_{f}$ is an affine Stanley symmetric function. In particular, given an ordinary permutation $w$ one can find an appropriate affine permutation $f_{w}$ with the property that $\tilde{F}_{f_{w}}=F_{w}$, giving some irreducible varieties $\Pi_{f_{w}}$. We prove Theorem 5.4 by degenerating $\Pi_{f_{w}}$ to a variety containing $X_{D(w)}$ as a top-dimensional component.

In fact, Stanley symmetric functions appear as the cohomology classes of a larger and more natural collection of positroid varieties. One nice way to describe positroid varieties is that they are exactly the projections of Richardson varieties (intersections of Schubert and opposite Schubert varieties) in the complete flag variety $\mathrm{Fl}(n)$ to $\operatorname{Gr}(k, n)$. Billey and Coskun (2012) considered the projections of Richardson varieties in the partial flag variety $\mathrm{Fl}\left(1, \ldots, k ; \mathbb{C}^{n}\right)$ to $\operatorname{Gr}(k, n)$, calling these rank varieties. Every rank variety is a positroid variety, but not vice versa.

Coskun (2009) gave a recursive rule for computing the cohomology class of a rank variety. We give a different formula for this class, in terms of ordinary Stanley symmetric functions.

Theorem 1.5 If $X \subseteq \operatorname{Gr}(k, n)$ is a rank variety, then $[X]=\phi\left(F_{w}\right)$ for some ordinary permutation $w$.

We will give a precise algorithm for finding $w$ from some combinatorial data defining $X$. We note that Theorem 1.5 (Theorem 4.1 below) is perhaps subtler than it looks in light of Knutson-Lam-Speyer's result that $[X]=\phi\left(\tilde{F}_{f}\right)$ for some affine permutation $f$. The issue is that $\tilde{F}_{f}$ need not be equal to any $F_{w}$; in fact, it may not even be Schur-positive (cf. Example 4.4). The subtle part of Theorem 1.5 is then that this issue lives in the kernel of $\phi$.

\section{A counterexample to Liu's conjecture}

We begin with a definition of the Specht modules $S^{D}$. Let $D$ be a diagram, with $|D|=m$. A bijective filling of $D$ is a bijection $T: D \rightarrow[m]$. The symmetric group $S_{m}$ acts on bijective fillings of $D$ by permuting entries. Fix a bijective filling $T$ of $D$. Let $R(T)$ denote the group of permutations $\sigma \in S_{m}$ for which $i, \sigma(i)$ are always in the same row of $T$. Let $C(T)$ be the analogous subgroup with "row" replaced by "column".

Definition 2.1 The Specht module of $D$ is the left ideal

$$
S^{D}=\mathbb{C}\left[S_{m}\right] \sum_{p \in R(T)} \sum_{q \in C(T)} \operatorname{sgn}(q) q p
$$

of $\mathbb{C}\left[S_{m}\right]$, viewed as an $S_{m}$-module. 
As $D$ runs over (Young diagrams of) partitions of $m$, the Specht modules provide a complete, irredundant set of complex irreducibles for $S_{m}$; more about these classical Specht modules can be found in Sagan (2001) or Fulton (1997). It is easy to show that the isomorphism type of $S^{D}$ does not depend on the choice of $T$, and that it is unaltered by permuting the rows or the columns of $D$. If the rows and columns of $D$ cannot be permuted to obtain a partition (equivalently, the rows of $D$ are not totally ordered under inclusion), then $S^{D}$ will not be irreducible.

In general it is an open problem to give a combinatorial rule for decomposing $S^{D}$ into irreducibles. The widest class of diagrams for which such a rule is known are the percent-avoiding diagrams, studied in Reiner and Shimozono (1998); see also Liu (2009) and Reiner and Shimozono (1995a). This class includes, for example, all skew diagrams, where the Littlewood-Richardson rule gives the decomposition. Liu's conjecture relates the Frobenius characteristic $s_{D}$ of $S^{D}$ to the diagram variety $X_{D}$ defined in Section 1

Conjecture 2 (Liu (2010)) For any diagram $D$, the cohomology classes $\left[X_{D}\right]$ and $\phi\left(s_{D}\right)$ are equal.

Liu proved Conjecture 2 in the case above where $D^{\vee}$ is a skew shape, or when it corresponds to a forest in the sense that one can represent a diagram $D \subset[k] \times[n-k]$ as the bipartite graph with white vertices $[k]$, black vertices $[n-k]$, and an edge between a white $i$ and black $j$ whenever $(i, j) \in D$. In Billey and Pawlowski (2014), we proved Conjecture 2 when $D^{\vee}$ is a permutation diagram and $S^{D}$ is multiplicity-free.

One gets a weaker version of Conjecture 2 by comparing degrees. The degree of a codimension $d$ subvariety $X$ of $\operatorname{Gr}(k, n)$ is the integer $\operatorname{deg}(X)$ defined by $[X] \sigma_{1}^{k(n-k)-d}=\operatorname{deg}(X) \sigma_{\left(k^{n-k}\right)}$. Under the Plücker embedding, this gives the usual notion of the degree of a subvariety of projective space, namely the number of points in the intersection of $X$ with a generic $d$-dimensional linear subspace. One can check using Pieri's rule that $\operatorname{deg}\left(\sigma_{\lambda}\right)=f^{\lambda^{\vee}}$, the number of standard Young tableaux of shape $\lambda^{\vee}$. This is also $\operatorname{dim} S^{\lambda^{\vee}}$. Since degree is additive on cohomology classes, Conjecture 2 predicts the following.

\section{Conjecture $3\left(\right.$ Liu (2010)) The degree of $X_{D}$ is $\operatorname{dim} S^{D^{\vee}}$.}

Liu proved Conjecture 3 when $D^{\vee}$ is a permutation diagram, and when $D^{\vee}$ has the property that if $\left(i, j_{1}\right),\left(i, j_{2}\right) \in D$ and $j_{1}<j<j_{2}$, then $(i, j) \in D$.

However, let us see that $D=\{(1,1),(2,2),(3,3),(4,4)\}$, with $k=4$ and $n=8$, is a counterexample to Conjectures 2 and 3 This is the skew diagram $4321 / 321$. The Specht module $S^{D}$ is simply the regular representation of $S_{4}$, with

$$
S^{D} \simeq S^{1111} \oplus 3 S^{211} \oplus 2 S^{22} \oplus 3 S^{31} \oplus S^{4} .
$$

It is a theorem of Magyar (1997) that the multiplicity of $S^{\lambda}$ in $S^{D}$ (for any $D$ ) is the multiplicity of $S^{\lambda^{\vee}}$ in $S^{D^{\vee}}$. Hence,

$$
S^{D^{\vee}} \simeq S^{3333} \oplus 3 S^{4332} \oplus 2 S^{4422} \oplus 3 S^{4431} \oplus S^{444},
$$

so $\operatorname{dim} S^{D^{\vee}}=f^{3333}+3 f^{4332}+2 f^{4422}+3 f^{4431}+f^{444}=24024$.

On the other hand, an explicit calculation in Macaulay2 shows $\operatorname{deg} X_{D}=21384$. Therefore Conjectures 3 and 2 both fail for $D$. (One may wonder how such a seemingly small counterexample remained undetected. It is really more natural to consider $D^{\vee}$ here-notice that the cases mentioned above for which Conjecture 2 has been established all have the property that $D^{\vee}$, rather than $D$, falls into some nice class of diagrams - and from this point of view the counterexample is no longer so small.) 
The discrepancy in degrees is $24024-21384=2640=f^{4422}$, which hints at how to see this discrepancy more explicitly. Given a $k$-subset $I$ of $[n]$, write $p_{I}$ for the corresponding Plücker coordinate on $\operatorname{Gr}(k, n)$, so $p_{I}(A)$ is the minor of $A$ in columns $I$. Let $Y$ be the scheme determined by the vanishing of the Plücker coordinates $p_{1678}, p_{2578}, p_{3568}, p_{4567}$. These are exactly the Plücker coordinates which vanish on $X_{D}$. One can check by computer that $\operatorname{codim} Y=4$, and so $Y$ is a complete intersection. This implies that

$$
[Y]=\sigma_{1}^{4}=\sigma_{1111}+3 \sigma_{211}+2 \sigma_{22}+3 \sigma_{31}+\sigma_{4},
$$

the cohomology class predicted for $\left[X_{D}\right]$ by Conjecture 2, see (Eisenbud and Harris, 2012, Section 5.2.1).

Since the four Plücker coordinates cutting out $Y$ vanish on $X_{D}^{\circ}$, the diagram variety $X_{D}$ is contained in $Y$. However, $Y$ has another top-dimensional component. The Plücker coordinates $p_{1678}, p_{2578}, p_{3568}, p_{4567}$ vanish on any matrix whose last four columns together have rank 2 or less. The locus of 4-planes represented by such matrices is the Schubert variety which is the closure of

$$
\left[\begin{array}{llllllll}
* & * & 1 & 0 & 0 & 0 & 0 & 0 \\
* & * & 0 & 1 & 0 & 0 & 0 & 0 \\
* & * & 0 & 0 & * & * & 1 & 0 \\
* & * & 0 & 0 & * & * & 0 & 1
\end{array}\right] .
$$

This Schubert variety has codimension 4 and degree $\operatorname{dim} S^{(22)^{\vee}}=f^{4422}=2640$, which is exactly $\operatorname{deg} Y-\operatorname{deg} X_{D}$. Therefore

$$
\left[X_{D}\right]=[Y]-\sigma_{22}=\sigma_{1111}+3 \sigma_{211}+\sigma_{22}+3 \sigma_{31}+\sigma_{4} .
$$

More counterexamples to Conjecture 2 can be easily manufactured from this one. For two diagrams $D_{1}$ and $D_{2}$ where $D_{1} \subseteq[a] \times[b]$, define $D_{1} \cdot D_{2}=D_{1} \cup\left\{(i+a, j+b):(i, j) \in D_{2}\right\}$. Graphically, $D_{1} \cdot D_{2}$ is the diagram

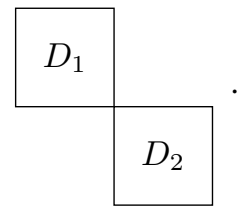

One can show that $\left[X_{D_{1} \cdot D_{2}}\right]=\left[X_{D_{1}}\right]\left[X_{D_{2}}\right]$ and similarly that $s_{D_{1} \cdot D_{2}}=s_{D_{1}} s_{D_{2}}$. Therefore if Conjecture 2 holds for $D_{1}$ but not $D_{2}$, then it will fail for $D_{1} \cdot D_{2}$.

Remark 2.2 One might naturally wonder whether a similar argument will show that Conjecture 2 fails for $D^{\prime}=\{(1,1),(2,2),(3,3),(4,4),(5,5)\}$. However, trying to repeat the analysis above runs into an immediate problem: the analogue of $Y$, which is the scheme $Z$ cut out by $p_{1789(10)}, p_{2689(10)}, p_{3679(10)}$, $p_{4678(10)}$, $p_{56789}$, no longer even has the same codimension as $X_{D}$ (thanks to Ricky Liu for pointing this out). Indeed, $X_{D}$ has codimension 5 but $Z$ contains the codimension 4 Schubert cell made up of rowspans of matrices whose last 5 columns have rank at most 3.

\section{Positroid varieties and rank varieties}

Definition 3.1 An affine permutation is a bijection $f: \mathbb{Z} \rightarrow \mathbb{Z}$ such that $f(i+n)=f(i)+n$ for all $i$ and some fixed $n$. Write $\breve{S}_{n}$ for the set of affine permutations with a particular $n$. 
The image of any set $\{a, a+1, \ldots, a+n-1\}$ completely determines an affine permutation. Call such an image a window. We will write affine permutations in one-line notation as the image of $[n]: 14825$ fixes 1 , sends 3 to 8,7 to 9 , etc. Members of any window are all distinct modulo $n$, so $\sum_{i=1}^{n} f(i) \equiv n(n+1) / 2$ $(\bmod n)$. Let $\operatorname{av}(f)$ be the integer $\frac{1}{n} \sum_{i=1}^{n} f(i)-i$.

Affine permutations are usually required to satisfy $\operatorname{av}(f)=0$, which ours need not. However, for a fixed $k$, affine permutations in $\breve{S}_{n}$ satisfying $\operatorname{av}(f)=k$ are in bijection with those satisfying av $(f)=0$ by subtracting $k$ from each entry in a window. When we refer to constructions on affine permutations that require a Coxeter group structure (e.g. length, reduced words, Stanley symmetric functions), we are implicitly using this isomorphism to transport that structure from the "usual" affine permutation group $\left\{f \in \breve{S}_{n}: \operatorname{av}(f)=0\right\}$.

Definition 3.2 An affine permutation $f \in \breve{S}_{n}$ is bounded if $i \leq f(i) \leq i+n$ for all $i$. Let $\operatorname{Bound}(k, n)$ denote the set of bounded affine permutations in $\breve{S}_{n}$ with $\operatorname{av}(f)=k$.

Any affine permutation $f$ has a permutation matrix, the $\mathbb{Z} \times \mathbb{Z}$ matrix $A$ with $A_{i, f(i)}=1$ and all other entries 0 . For any $i, j \in \mathbb{Z}$, define

$$
[i, j](f)=\{p<i: f(p)>j\}
$$

Thus, $\#[i, j](f)$ is the number of 1's strictly northeast of $(i, j)$ in the permutation matrix of $f$, in matrix coordinates.

Fix a basis $e_{1}, \ldots, e_{n}$ of $\mathbb{C}^{n}$. We will abuse notation by writing $\langle X\rangle$ both for the span of the vectors in $X$, if $X \subseteq \mathbb{C}^{n}$, and for the span of vectors $e_{i}$ with $i \in X$, if $X \subseteq[n]$. If $X \subseteq[n]$, let $\operatorname{Proj}_{X}: \mathbb{C}^{n} \rightarrow\langle X\rangle$ be the projection which fixes those basis vectors $e_{i}$ with $i \in X$ and sends the rest to 0 . For integers $i \leq j$, write $[i, j]$ for $\{i, i+1, \ldots, j\}$. We interpret indices of basis vectors modulo $n$, so that $\langle[i, j]\rangle \subseteq \mathbb{C}^{n}$ even if $i, j$ fail to lie in $[1, n]$.

Definition 3.3 (Knutson et al. (2013)) Given a bounded affine permutation $f \in \operatorname{Bound}(k, n)$, the positroid variety $\Pi_{f} \subseteq \operatorname{Gr}(k, n)$ is the closure of

$$
\left\{V \in \operatorname{Gr}(k, n): \operatorname{dim}\left(\operatorname{Proj}_{[i, j]} V\right)=k-\#[i, j](f) \text { for all } i \leq j\right\} .
$$

Theorem 3.4 (Knutson et al. (2013), Theorem 5.9) The positroid variety $\Pi_{f} \subseteq \operatorname{Gr}(k, n)$ is irreducible of codimension $\ell(f)$.

Here the length $\ell(f)$ of an affine permutation $f$ is the number of inversions $i<j, f(i)>f(j)$, provided that we regard any two inversions $i<j$ and $i+p n<j+p n$ as equivalent.

Knutson-Lam-Speyer also computed the cohomology class of $\Pi_{f}$ in terms of the affine Stanley symmetric function $\tilde{F}_{f}$. These are a class of symmetric functions indexed by affine permutations introduced in Lam (2006), which can be thought of as generating functions for certain factorizations of reduced words for an affine permutation.

Theorem 3.5 (Knutson et al. (2013), Theorem 7.1) For $f \in \operatorname{Bound}(k, n)$, the cohomology class $\left[\Pi_{f}\right]$ is $\phi\left(\tilde{F}_{f}\right)$.

The ordinary Stanley symetric functions indexed by members of $S_{n}$, introduced in Stanley (1984), are a special case of affine Stanley symmetric functions. To be precise, we can view $w \in S_{n}$ as the affine permutation in $\breve{S}_{n}^{0}$ sending $i+p n$ to $w(i)+p n$ for $1 \leq i \leq n$. Then the Stanley symmetric function $F_{w}$ 
of $w$ is $\tilde{F}_{w}$. This is Proposition 5 in Lam (2006), but we will simply take it as a definition of $F_{w}$. One should be aware, however, that the $F_{w}$ defined in Stanley (1984) is our $F_{w^{-1}}$.

Now we discuss a subset of positroid varieties whose cohomology classes will turn out, in Section 4 to be represented by ordinary Stanley symmetric functions.

Definition 3.6 (Billey and Coskun (2012)) $A$ rank set is a finite set of intervals $M=\left\{\left[a_{1}, b_{1}\right], \ldots,\left[a_{k}, b_{k}\right]\right\}$ with $a_{i} \leq b_{i}$ positive integers, where all $a_{i}$ are distinct and all $b_{i}$ are distinct.

Suppose $M$ is a rank set with $b \leq n$ for all $[a, b] \in M$, and $\# M=k$. If $S$ is a set of positive integers, let $S(M)$ denote the set of intervals $S^{\prime} \in M$ such that $S^{\prime} \subseteq S$. Coskun (2009) defines a closed subvariety $\Sigma_{M}$ of $\operatorname{Gr}(k, n)$ as the closure of the locus

$$
\{V \in \operatorname{Gr}(k, n): \operatorname{dim}(V \cap\langle S \cap T\rangle)=\#(S \cap T)(M) \text { for } S, T \in M\} .
$$

$\Sigma_{M}$ is called a rank variety.

Theorem 3.7 (Coskun (2009), Lemma 3.29) The rank variety $\Sigma_{M}$ is irreducible of dimension $\sum_{S \in M}(\# S-$ $\# S(M))$.

The variety $\Sigma_{M}$ has a useful interpretation in coordinates.

Lemma 3.8 Let $U_{M}$ be the locus of $k$-planes with a basis $\left\{v_{S}: S \in M\right\}$, indexed by the intervals in $M$, such that the coefficient of $e_{i}$ in $v_{S}$ is nonzero if and only if $i \in S$. Then $\overline{U_{M}}=\Sigma_{M}$.

Example 3.9 If $M=\{[1,2],[3,4],[2,5]\}$ and $n=5$, then

$$
U_{M}=\left\{\operatorname{rowspan}\left(\begin{array}{ccccc}
* & * & 0 & 0 & 0 \\
0 & 0 & * & * & 0 \\
0 & * & * & * & *
\end{array}\right): \text { every } * \text { nonzero }\right\}=\left\{\operatorname{rowspan}\left(\begin{array}{lllll}
* & 1 & 0 & 0 & 0 \\
0 & 0 & * & 1 & 0 \\
0 & * & * & 0 & 1
\end{array}\right)\right\}
$$

Being defined by rank conditions on intersections with interval subspaces, rank varieties should be special instances of positroid varieties. Say $M=\left\{\left[a_{1}, b_{1}\right], \ldots,\left[a_{k}, b_{k}\right]\right\}$ is a rank set with $b_{1}<\cdots<$ $b_{k} \leq n$. Define

$$
\begin{aligned}
\left\{c_{1}<\cdots<c_{n-k}\right\} & =[n] \backslash\left\{a_{1}, \ldots, a_{k}\right\} \text { and } \\
\left\{d_{1}<\cdots<d_{n-k}\right\} & =[n] \backslash\left\{b_{1}, \ldots, b_{k}\right\} .
\end{aligned}
$$

Let $f_{M}$ be the affine permutation mapping $b_{i}$ to $a_{i}+n$ and $d_{i}$ to $c_{i}$. Then $f_{M}$ is bounded because $a_{i} \leq b_{i}$, which implies $d_{i} \leq c_{i}$. This provides a bijection between rank sets for $\operatorname{Gr}(k, n)$ and members $f$ of $\operatorname{Bound}(k, n)$ such that the subsequence of $f(1) \cdots f(n)$ with entries in $[n]$ is increasing.

Example 3.10 Take $M=\{[1,1],[3,4],[2,5]\}$ and $n=5$ as above. Then $b_{1}=1, b_{2}=4, b_{3}=5$ and $a_{1}=1, a_{2}=3, a_{3}=2$, so $d_{1}=2, d_{2}=3$ while $c_{1}=4, c_{2}=5$. Hence $f_{M}=64587$.

Theorem 3.11 The rank variety $\Sigma_{M}$ is the positroid variety $\Pi_{f_{M}}$. 


\section{Cohomology classes of rank varieties}

Let $\Lambda$ be the ring of symmetric functions over $\mathbb{Z}$, and $\phi: \Lambda \rightarrow H^{*}(\operatorname{Gr}(k, n), \mathbb{Z})$ the ring homomorphism sending the Schur function $s_{\lambda}$ to the Schubert class $\sigma_{\lambda}$, or to 0 if $\lambda$ is not contained in a $k \times(n-k)$ rectangle.

Coskun (2009) gives a recursive rule to calculate the cohomology class of a rank variety. Since rank varieties are positroid varieties, Theorem 3.5 gives a more direct answer, namely that $\left[\Sigma_{M}\right]$ is $\phi\left(\tilde{F}_{f_{M}}\right)$. The goal of this section is to show that $\left[\bar{\Sigma}_{M}\right]$ is actually represented by an ordinary Stanley symmetric function.

Theorem 4.1 For any rank variety $\Sigma_{M} \subseteq \operatorname{Gr}(k, n)$, there is a permutation $w_{M}$ such that $\left[\Sigma_{M}\right]=\phi\left(F_{w}\right)$.

Our strategy will be to replace a rank set $M$ with a new rank set $M^{\prime}$ in such a way that the truth of Theorem 4.1 for $\Sigma_{M^{\prime}}$ implies it for $\Sigma_{M}$, and so that after enough replacements we end up with a rank variety $\Sigma_{N}=\Pi_{f}$ where $\tilde{F}_{f}$ is obviously an ordinary Stanley symmetric function.

Specifically, if $M$ is a rank set, define a new rank set $\kappa(M)=\{[a, b+1]:[a, b] \in M\}$. We call the operation $\kappa$ stretching. Say that $M$ is stretched if every right endpoint is right of every left endpoint; that is, whenever $S, T \in M$, we have $\min (S)<\max (T)$. For any $M$, there is an $m$ so that $\kappa^{m}(M)$ is stretched. Given a rank variety $\Sigma_{M}$ in $\operatorname{Gr}(k, n)$, we always interpret $\Sigma_{\kappa M}$ as a subvariety of $\operatorname{Gr}(k, n+1)$. Let $\tau$ be the affine permutation $\tau(i)=i+1$.

Lemma 4.2 Suppose $M$ is stretched and $\Sigma_{M} \subseteq \operatorname{Gr}(k, n)$. Let $b$ be minimal such that $[a, b] \in M$ for some $a$, and let $y=f_{M}(b-1)$. Then $\tau^{-y+1} f_{M} \tau^{b-2}$ restricted to $[n]$ is an ordinary permutation $w \in S_{n}$, and $\tilde{F}_{f_{M}}=\phi\left(F_{w}\right)$.

We now give a precise relationship between the classes of $\Sigma_{M}$ and $\Sigma_{\kappa M}$. Given a map $\iota: \operatorname{Gr}(k, n) \rightarrow$ $\operatorname{Gr}(k, n+1)$, we get a pullback $\iota^{*}: H^{p}(\operatorname{Gr}(k, n+1), \mathbb{Z}) \rightarrow H^{p}(\operatorname{Gr}(k, n), \mathbb{Z})$.

Theorem 4.3 Let $\iota: \operatorname{Gr}(k, n) \hookrightarrow \operatorname{Gr}(k, n+1)$ be the inclusion induced by a linear inclusion $\mathbb{C}^{n} \hookrightarrow$ $\mathbb{C}^{n+1}$. Then $\iota^{*}\left[\Sigma_{\kappa M}\right]=\left[\Sigma_{M}\right]$.

The pullback $\iota^{*}$ of such an inclusion sends $\sigma_{\lambda}$ to $\sigma_{\lambda}$ if $\lambda \subseteq[k] \times[n-k]$ and to 0 otherwise. Thus if $\left[\Sigma_{\kappa M}\right]=\phi(g)$ for some symmetric function $g$, then $\left[\Sigma_{M}\right]=\phi(g)$ as well (here we are abusing notation since these two instances of $\phi$ are really different maps). In particular, if we iterate Theorem 4.3 until $\kappa^{m} M$ is stretched, then we have $\left[\Sigma_{\kappa^{m}} M\right]=\phi\left(F_{w}\right)$ for some ordinary permutation $w$ by Lemma 4.2 , and hence $\left[\Sigma_{M}\right]=\phi\left(F_{w}\right)$, proving Theorem 4.1 .

This argument gives a simple algorithm for taking a rank variety $\Sigma_{M} \subseteq \operatorname{Gr}(k, n)$ and producing a permutation $w_{M}$ such that $\left[\Sigma_{M}\right]=\phi\left(F_{w_{M}}\right)$.

Step 1. Choose $m$ such that $\kappa^{m} M$ is stretched.

Step 2. Find $b$ minimal such that $[a, b] \in \kappa^{m} M$, and set $y=f_{\kappa^{m} M}(b-1)$.

Step 3. Define $w_{M} \in S_{n}$ by $w_{M}(i)=f_{\kappa^{m} M}(b-2+i)-y+1$.

Example 4.4 Let $M=\{[1,1],[3,3]\}$ in $\operatorname{Gr}(2,4)$, so $\Sigma_{M}$ has a dense open subset consisting of rowspans of matrices with the form

$$
\left[\begin{array}{cccc}
* & 0 & 0 & 0 \\
0 & 0 & * & 0
\end{array}\right]
$$


That is, $\Sigma_{M}$ is the single point $\left\langle e_{1}, e_{3}\right\rangle$. The minimal $m$ such that $\kappa^{m} M$ is stretched is $m=3$, and $\kappa^{3} M=\{[1,4],[3,7]\}$. We have $f_{M}=5274$ and $f_{\kappa^{3} M}=24586(10) 7$. The minimal $b$ with $[a, b] \in \kappa^{2} M$ is $b=4$, and then $y=f_{\kappa^{2} M}(b-1)=5$.

Write out more entries of $f_{\kappa^{2} M}$, demarcating the window from 1 to $n+m=7$ with vertical bars and denoting negative numbers with horizontal bars:

$$
f_{\kappa^{2} M}=\cdots \overline{5} \overline{3} \overline{2} 1 \overline{1} 30|24586(10) 7| 9(11)(12)(15)(13)(17)(14) \cdots
$$

Shift the window so that its leftmost entry is in position $b-1=3$ :

$$
f_{\kappa^{2} M}=\cdots \overline{5} \overline{3} \overline{2} 1 \overline{1} 3024|586(10) 79(11)|(12)(15)(13)(17)(14) \cdots
$$

The entries of the window now fill out exactly the interval [5, 11]. That is, $\tau^{-4} f_{\kappa^{3} M} \tau^{2}$ restricted to [7] is an ordinary permutation, namely $w_{M}=1426357$. Indeed, $\left[\Sigma_{M}\right]=\sigma_{22}$ is the class of a point, while $\tilde{F}_{f_{M}}=s_{22}+s_{31}-s_{4}$ and $F_{w_{M}}=s_{22}+s_{31}$.

\section{Degenerations of rank varieties}

Let $\phi_{t, i \rightarrow j}$ be the linear transformation sending $e_{i}$ to $t e_{i}+(1-t) e_{j}$. For $t \neq 0$, the varieties $\phi_{t, i \rightarrow j} \Sigma_{M}$ are all isomorphic, so they form a flat family (Eisenbud and Harris, 2000, Proposition III-56). The flat limit $\lim _{t \rightarrow 0} \phi_{t, i \rightarrow j} \Sigma_{M}$ then exists as a scheme (Hartshorne, 1977, Proposition 9.8). The key fact for us is that $\Sigma_{M}$ and $\lim _{t \rightarrow 0} \phi_{t, i \rightarrow j} \Sigma_{M}$ have the same Chow ring class, hence the same cohomology class. In this section we will show that for an appropriate choice of $M, \lim _{t \rightarrow 0} \phi_{t, i \rightarrow j} \Sigma_{M}$ contains the diagram variety $X_{D(w)}$ as an irreducible component. Other authors have used these degenerations or similar ones to calculate cohomology classes or K-theory classes of subvarieties of Grassmannian, including Coskun (2009) and Vakil (2006).

Define an operator $C_{i \rightarrow j}$ on matrices of a fixed size as follows:

$$
\left(C_{i \rightarrow j} A\right)_{p q}= \begin{cases}A_{p i} & \text { if } q=j \text { and } A_{p j}=0 \\ 0 & \text { if } q=i \text { and } A_{p j}=0 \\ A_{p q} & \text { otherwise. }\end{cases}
$$

For example,

$$
C_{1 \rightarrow 2}\left[\begin{array}{cc}
1 & 0 \\
0 & 2 \\
3 & -7 \\
0 & 0
\end{array}\right]=\left[\begin{array}{cc}
0 & 1 \\
0 & 2 \\
3 & -7 \\
0 & 0
\end{array}\right]
$$

Sometimes we also apply $C_{i \rightarrow j}$ to $k$-planes, or sets of $k$-planes. Strictly speaking this is ill-defined, since it can happen that rowspan $A=\operatorname{rowspan} A^{\prime}$ but rowspan $C_{i \rightarrow j} A \neq \operatorname{rowspan} C_{i \rightarrow j} A^{\prime}$, so we only use this notation when the $k$-planes are represented by specific matrices.

Lemma 5.1 Say $F$ is any subset of $[k] \times[n-k]$ and $U$ is the set of $k$-planes rowspan $(A)$ where $A_{i j}=0$ whenever $(i, j) \in F$. If rowspan $A \in U$ and $C_{i \rightarrow j} A$ has rank $k$, then rowspan $C_{i \rightarrow j} A \in$ $\lim _{t \rightarrow 0} \phi_{t, i \rightarrow j} \bar{U}$. 
Rank varieties and diagram varieties both have dense open subsets to which Lemma 5.1 applies. We will apply this lemma to rank varieties, but it has an interesting interpretation for diagram varieties as well. Define $C_{i \rightarrow j}$ on diagrams as on matrices (e.g. by viewing diagrams as 0,1-matrices). Lemma 5.1 shows that $X_{C_{i \rightarrow j} D} \subseteq \lim _{t \rightarrow 0} \phi_{t, i \rightarrow j} X_{D}$, which implies that $\left[X_{D}\right]-\left[X_{C_{i \rightarrow j} D}\right]$ is a nonnegative linear combination of Schubert classes. On the other hand, James and Peel (1979) showed that for the diagram Specht modules $S^{D}$, there is always an $S_{|D|}$-equivariant injection $S^{C_{i \rightarrow j} D} \hookrightarrow S^{D}$. Equivalently, $s_{D}-s_{C_{i \rightarrow j} D}$ is a nonnegative linear combination of Schur functions. A more powerful version of this connection is important in Liu's proofs of several cases of Conjectures 2 and 3 in Liu (2010).

Given a permutation $w \in S_{n}$, define a rank set $M(w)=\{[w(i), i+n]: 1 \leq i \leq n\}$, so $\Sigma_{M(w)} \subseteq$ $\operatorname{Gr}(n, 2 n)$. Then

$$
f_{M(w)}=(n+1) \cdots(2 n)(w(1)+2 n) \cdots(w(n)+2 n)=(w \times 12 \cdots n) \tau^{-n} .
$$

Here, for $w \in S_{n}$ and $v \in S_{m}, w \times v$ is the permutation in $S_{n+m}$ sending $i$ to $w(i)$ if $i \leq n$ and to $v(i-n)+n$ otherwise. Thus $\tilde{F}_{f_{M(w)}}=\tilde{F}_{w \times 12 \cdots n}=F_{w \times 12 \cdots n}=F_{w}$. This shows that $\left[\Sigma_{M(w)}\right]=\phi\left(F_{w}\right)$.

Define

$$
\begin{aligned}
& C_{w}=C_{n+1 \rightarrow w(1)} \circ C_{n+2 \rightarrow w(2)} \circ \cdots \circ C_{2 n \rightarrow w(n)} \\
& \phi_{t, w}=\phi_{t, n+1 \rightarrow w(1)} \circ \phi_{t, n+2 \rightarrow w(2)} \circ \cdots \circ \phi_{t, 2 n \rightarrow w(n)} .
\end{aligned}
$$

We will show that $\lim _{t \rightarrow 0} \phi_{t, w} \Sigma_{M(w)}$ contains the diagram variety $X_{D(w)}$ as an irreducible component. First we give an explicit example of this degeneration.

Example 5.2 Take $n=5$ and $w=24153$, so $M(w)=\{[2,6],[4,7],[1,8],[5,9],[3,10]\}$. Then $\Sigma_{M(w)}$ is the closure of

$$
\left.\left\{\begin{array}{llllllllll}
0 & * & * & * & * & * & 0 & 0 & 0 & 0 \\
0 & 0 & 0 & * & * & * & * & 0 & 0 & 0 \\
* & * & * & * & * & * & * & * & 0 & 0 \\
0 & 0 & 0 & 0 & * & * & * & * & * & 0 \\
0 & 0 & * & * & * & * & * & * & * & *
\end{array}\right)\right\}
$$

Here $C_{w}=C_{6 \rightarrow 2} C_{7 \rightarrow 4} C_{8 \rightarrow 1} C_{9 \rightarrow 5} C_{10 \rightarrow 3}$, and

$$
C_{w} \Sigma_{M(w)} \supseteq\left\{\operatorname{rowspan}\left(\begin{array}{cccccccccc}
0 & * & * & * & * & * & 0 & 0 & 0 & 0 \\
0 & * & 0 & * & * & 0 & * & 0 & 0 & 0 \\
* & * & * & * & * & * & * & * & 0 & 0 \\
* & * & 0 & * & * & 0 & 0 & 0 & * & 0 \\
* & * & * & * & * & 0 & * & 0 & * & *
\end{array}\right)\right\} .
$$

Notice that the 0 's forced in columns 1 through 5 of these matrices form the diagram D(24153). Moreover, on the open set where columns 6 through 10 are invertible, we can clear out the underlined entries below the diagonal without affecting the pattern of 0 's and *'s in columns 1 through 5 . Hence,

$$
C_{w} \Sigma_{M(w)} \supseteq\left\{\operatorname{rowspan}\left(\begin{array}{cccccccccc}
0 & * & * & * & * & 1 & 0 & 0 & 0 & 0 \\
0 & * & 0 & * & * & 0 & 1 & 0 & 0 & 0 \\
* & * & * & * & * & 0 & 0 & 1 & 0 & 0 \\
* & * & 0 & * & * & 0 & 0 & 0 & 1 & 0 \\
* & * & * & * & * & 0 & 0 & 0 & 0 & 1
\end{array}\right)\right\}=X_{D(24153)}^{\circ} .
$$


By Lemma 5.1. this shows that $X_{D(24153)} \subseteq \lim _{t \rightarrow 0} \phi_{t, M} \Sigma_{M(w)}$.

The argument of Example 5.2 works in general.

Theorem 5.3 The flat limit $\lim _{t \rightarrow 0} \phi_{t, M} \Sigma_{M(w)}$ contains $X_{D(w)}$ as an irreducible component.

Since flat limits preserve cohomology classes, and $\left[\Sigma_{M(w)}\right]=\phi\left(F_{w}\right)$, the next theorem is an immediate corollary.

Theorem $5.4 \phi\left(F_{w}\right)-X_{D(w)}$ is a nonnegative combination of Schubert classes.

However, this difference of classes can be nonzero. Indeed, the counterexample $D=\{(1,1),(2,2),(3,3),(4,4)\}$ to Conjecture 2 discussed in Section 2 provides an example. Take $w=21436587$. Then $D(w)=$ $\{(1,1),(3,3),(5,5),(7,7)\}$ can be obtained from $D$ by permuting rows and columns, and viewing $D$ in a larger rectangle. Neither of these operations on diagrams affects $s_{D}$ or $\left[X_{D}\right]$, identifying the latter with its pullback along a linear embedding of $\operatorname{Gr}(k, n)$ into $\operatorname{Gr}(k, n+1)$ or $\operatorname{Gr}(k+1, n+1)$. Thus Conjecture 2 can fail for permutation diagrams.

\section{Acknowledgements}

I would like to thank Dave Anderson, Andy Berget, Sara Billey, Izzet Coskun, Ricky Liu, Vic Reiner, and Robert Smith for many helpful comments and discussions.

\section{References}

S. Billey and I. Coskun. Singularities of generalized Richardson varieties. Comm. Algebra, 40(4):14661495, 2012.

S. Billey and B. Pawlowski. Permutation patterns, Stanley symmetric functions, and generalized Specht modules. Journal of Combinatorial Theory Series A, 127:85-120, 2014.

I. Coskun. A Littlewood-Richardson rule for two-step flag varieties. Invent. Math., 176(2):325-395, 2009.

D. Eisenbud and J. Harris. The Geometry of Schemes. Springer-Verlag, 2000.

D. Eisenbud and J. Harris. $3264 \&$ all that : Intersection theory in algebraic geometry. Draft available at http://isites.harvard.edu/fs/docs/icb.topic720403.files/book.pdf, 2012.

W. Fulton. Young Tableaux: With Applications to Representation Theory and Geometry. Cambridge University Press, 1997.

R. Hartshorne. Algebraic Geometry. Springer-Verlag, 1977.

G. James and M. Peel. Specht series for skew representations of symmetric groups. Journal of Algebra, 56:343-364, 1979.

A. Knutson, T. Lam, and D. Speyer. Positroid varieties: Juggling and geometry. Compos. Math., 149: 1710-1752, 2013. 
W. Kraśkiewicz and P. Pragacz. Schubert functors and Schubert polynomials. European Journal of Combinatorics, 25:1327-1344, 2004.

T. Lam. Affine Stanley symmetric functions. Amer. J. Math., 128:1553-1586, 2006.

R. Liu. Matching polytopes and Specht modules. Transactions of the American Mathematical Society, 364:1089-1107, 2009.

R. Liu. Specht Modules and Schubert Varieties for General Diagrams. PhD thesis, Massachusetts Institute of Technology, 2010.

P. Magyar. Borel-Weil theorem for Configuration Varieties and Schur Modules. Advances in Mathematics, 134(2):328-366, 1997.

A. Postnikov. Total positivity, Grassmannians, and networks. arXiv:math/0609764, 2006.

V. Reiner and M. Shimozono. Specht series for column-convex diagrams. Journal of Algebra, 174: 489-522, 1995a.

V. Reiner and M. Shimozono. Plactification. Journal of Algebraic Combinatorics, 4:331-351, $1995 \mathrm{~b}$.

V. Reiner and M. Shimozono. Percentage-avoiding, northwest shapes, and peelable tableaux. Journal of Combinatorial Theory, Series A, 82:1-73, 1998.

B. Sagan. The Symmetric Group: Representations, Combinatorial Algorithms, and Symmetric Functions. Springer-Verlag, 2001.

R. P. Stanley. On the number of reduced decompositions of elements of Coxeter groups. European Journal of Combinatorics, 5:359-372, 1984.

R. Vakil. A geometric Littlewood-Richardson rule. Annals of Mathematics, 164(2):371-421, 2006. 